\title{
WEIGHTED INEQUALITIES AND STEIN-WEISS POTENTIALS
}

\author{
WILLIAM BECKNER
}

\begin{abstract}
Sharp extensions of Pitt's inequality and bounds for Stein-Weiss fractional integrals are obtained that incorporate gradient forms and vector-valued operators. Such results include HardyRellich inequalities.
\end{abstract}

Weighted inequalities provide quantitative information to characterize integrability for differential and integral operators and intrinsically are determined by their dilation character. In the classical context, weighted inequalities for the Fourier transform provide a natural measure of uncertainty. For functions on $\mathbb{R}^{n}$ the issue is the balance between the relative size of a function and its Fourier transform at infinity. An inequality that illustrates this principle at the spectral level is Pitt's inequality:

$$
\int_{\mathbb{R}^{n}} \Phi(1 /|x|)|f(x)|^{2} d x \leq C_{\Phi} \int_{\mathbb{R}^{n}} \Phi(|y|)|\widehat{f}(y)|^{2} d y
$$

where $\Phi$ is an increasing function, the function $f$ is in the Schwartz class $\mathcal{S}\left(\mathbb{R}^{n}\right)$ and the Fourier transform is defined by

$$
(\mathcal{F} f)(y)=\widehat{f}(y)=\int_{\mathbb{R}^{n}} e^{2 \pi i x y} f(x) d x .
$$

Such inequalities may be fully determined by dilation invariance, and some cases may be realized with explicit gradient forms as Hardy-Rellich inequalities. In earlier work (see [3) the effective calculation for the constant in Pitt's inequality was reduced to Young's inequality for convolution on a non-compact unimodular group. The objective here will be to study more general forms of Pitt's inequality

$$
\int_{\mathbb{R}^{n}} \Phi(1 /|x|)|\nabla f|^{2} d x \leq 4 \pi^{2} D_{\Phi} \int_{\mathbb{R}^{n}} \Phi(|y|)|y|^{2}|\widehat{f}(y)|^{2} d y
$$

using the structure of Stein-Weiss potentials and convolution estimates in concert with the Hecke-Bochner representation for $L^{2}\left(\mathbb{R}^{n}\right)$. The previous work is described by the following three theorems.

Theorem 1 (Pitt's inequality). For $f \in \mathcal{S}\left(\mathbb{R}^{n}\right)$ and $0 \leq \alpha<n$

$$
\begin{gathered}
\int_{\mathbb{R}^{n}}|x|^{-\alpha}|f(x)|^{2} d x \leq C_{\alpha} \int_{\mathbb{R}^{n}}|y|^{\alpha}|\widehat{f}(y)|^{2} d y \\
C_{\alpha}=\pi^{\alpha}\left[\Gamma\left(\frac{n-\alpha}{4}\right) / \Gamma\left(\frac{n+\alpha}{4}\right)\right]^{2} .
\end{gathered}
$$

Since the above inequality becomes an identity for $\alpha=0$, a differentiation argument provides a logarithmic form that controls the uncertainty principle by using dimensional asymptotics.

Theorem 2 (logarithmic uncertainty). For $f \in \mathcal{S}\left(\mathbb{R}^{n}\right)$

$$
\begin{gathered}
\int_{\mathbb{R}^{n}} \ln |x||f(x)|^{2} d x+\int_{\mathbb{R}^{n}} \ln |y||\widehat{f}(y)|^{2} d y \geq D \int|f(x)|^{2} d x \\
D=\psi(n / 4)-\ln \pi, \quad \psi(t)=\frac{d}{d t} \ln \Gamma(t) .
\end{gathered}
$$

Logarithmic integrals are indeterminate so $D$ may take negative values. The proof of Pitt's inequality (3) follows from a sharp estimate for an equivalent integral realization as a Stein-Weiss fractional integral on $\mathbb{R}^{n}$. 
Theorem 3. For $f \in L^{2}\left(\mathbb{R}^{n}\right)$ and $0<\alpha<n$

$$
\begin{gathered}
\left|\int_{\mathbb{R}^{n} \times \mathbb{R}^{n}} f(x) \frac{1}{|x|^{\alpha / 2}} \frac{1}{|x-y|^{n-\alpha}} \frac{1}{|y|^{\alpha / 2}} f(y) d x d y\right| \leq B_{\alpha} \int_{\mathbb{R}^{n}}|f(x)|^{2} d x \\
B_{\alpha}=\pi^{n / 2}\left[\Gamma\left(\frac{\alpha}{2}\right) / \Gamma\left(\frac{n-\alpha}{2}\right)\right]\left[\Gamma\left(\frac{n-\alpha}{4}\right) / \Gamma\left(\frac{n+\alpha}{4}\right)\right]^{2} .
\end{gathered}
$$

The development of the sharp estimate for the Stein-Weiss integral rests on using symmetrization to reduce the problem to radial functions, and then as a consequence of dilation invariance the estimate can be converted to Young's inequality for convolution on the multiplicative group $\mathbb{R}_{+}$(or alternatively on $\mathbb{R})$.

$$
\|\varphi * f\|_{L^{2}(G)} \leq\|\varphi\|_{L^{1}(G)}\|f\|_{L^{2}(G)}
$$

When $\varphi$ is non-negative, the inequality is sharp with no extremal functions. One objective here will be to extend this inequality to cases when $\psi$ takes both positive and negative values by using the Hecke-Bochner formulas.

Since the form

$$
\int_{\mathbb{R}^{n}}|\widehat{f}(y)|^{2}|y|^{\alpha} d y=(2 \pi)^{-\alpha} \int_{\mathbb{R}^{n}}\left|(-\Delta)^{\alpha / 4} f\right|^{2} d x
$$

can be regarded in the family of gradient estimates, Pitt's inequality has been characterized as a HardyRellich inequality in some parts of the recent literature with alternative proofs and extensions (see [1], 7], [14, and [17). Some natural generalizations of Pitt's inequality can now be viewed in the context of the arguments developed in 3 where proofs of Theorems 1,2 and 3 are given.

\section{Pitt's inequality with gradient terms}

Theorem 4. For $f \in \mathcal{S}\left(\mathbb{R}^{n}\right)$ and $0<\alpha<n, n>1$

$$
\begin{gathered}
\int_{\mathbb{R}^{n}}|\nabla f|^{2}|x|^{-\alpha} d x \leq 4 \pi^{2} D_{\alpha} \int_{\mathbb{R}^{n}}|\widehat{f}(y)|^{2}|y|^{\alpha+2} d y \\
D_{\alpha}=\pi^{\alpha} \max _{k}\left\{\left[\Gamma\left(\frac{n+2 k-\alpha+2}{4}\right) / \Gamma\left(\frac{n+2 k+\alpha+2}{4}\right)\right]^{2}\left(1+\frac{4 k \alpha}{(n+2 k-\alpha-2)^{2}}\right)\right\}
\end{gathered}
$$

By convention the last term in the line above is one when $k=0$. This result is interesting for several aspects:

(a) for the gradient term with radial functions (i.e. $k=0$ ) the constant is reduced from that in equality (3) since $\Gamma(x+\beta) / \Gamma(y+\beta)$ is decreasing in $\beta$ for $x<y$; this reduction in constant is also apparent by the Plancherel theorem;

(b) the corresponding Stein-Weiss integral does not have a positive kernel though it does have symmetry in the angular variables;

(c) the limiting logarithmic uncertainty is sharper

$$
\begin{gathered}
\int_{\mathbb{R}^{n}} \ln |x||\nabla f|^{2} d x+\int_{\mathbb{R}^{n}} \ln |y|\left(4 \pi^{2}|y|^{2}\right)|\widehat{f}(y)|^{2} d y \geq E \int_{\mathbb{R}^{n}}|\nabla f|^{2} d x \\
E= \begin{cases}\psi\left(\frac{3}{2}\right)-\ln \pi-1, & n=2 \\
\psi\left(\frac{n}{4}+\frac{1}{2}\right)-\ln \pi, & n \geq 3\end{cases}
\end{gathered}
$$

(d) for $\alpha=2$ and $n>4$ one obtains the Hardy-Rellich inequality

$$
\int_{\mathbb{R}^{n}}|\nabla f|^{2}|x|^{-2} d x \leq \frac{4}{n^{2}} \int_{\mathbb{R}^{n}}|\Delta f|^{2} d x ;
$$

and (e) for some values of the parameters $n$ and $\alpha$, the sharp bound is obtained by considering non-radial functions. A sharper version of (77) appears in [14. 
By using the Fourier transform on Riesz potentials

$$
\mathcal{F}\left[|x|^{-\alpha}\right]=\pi^{-\frac{n}{2}+\alpha}\left[\Gamma\left(\frac{n-\alpha}{2}\right) / \Gamma\left(\frac{\alpha}{2}\right)\right]|x|^{-n+\alpha},
$$

one easily sees that Pitt's inequality in Theorem 4 is equivalent to a Stein-Weiss fractional integral inequality on $\mathbb{R}^{n}$.

Theorem 5. For $f \in L^{2}\left(\mathbb{R}^{n}\right)$ and $0<\alpha<n, n>1$

$$
\begin{gathered}
\left|\int_{\mathbb{R}^{n} \times \mathbb{R}^{n}} f(x) \frac{1}{|x|^{\alpha / 2}} \frac{x \cdot y}{|x||y|} \frac{1}{|x-y|^{n-\alpha}} \frac{1}{|y|^{\alpha / 2}} f(y) d x d y\right| \\
\leq\left[\pi^{\frac{n}{2}-\alpha} \Gamma\left(\frac{\alpha}{2}\right) / \Gamma\left(\frac{n-\alpha}{2}\right)\right] D_{\alpha} \int_{\mathbb{R}^{n}}|f|^{2} d x
\end{gathered}
$$

with $D_{\alpha}$ as in Theorem 4 .

Proof of Theorems 4 and 5 . The main difficulty with showing these estimates is that the Stein-Weiss kernel is not positive. This obstruction is addressed in two steps.

Step 1. Assume $f$ is radial and set $t=|x|, h(t)=|x|^{n / 2} f(x)$; then (8) reduces to

$$
\left|\int_{\mathbb{R}_{+} \times \mathbb{R}_{+}} h(t) \psi_{\alpha}(s / t) h(s) \frac{d s}{s} \frac{d s}{t}\right| \leq D_{\alpha}\left[\Gamma\left(\frac{n}{2}\right) \Gamma\left(\frac{\alpha}{2}\right) / 2 \pi^{\alpha} \Gamma\left(\frac{n-\alpha}{2}\right)\right] \int_{\mathbb{R}_{+}}|h|^{2} \frac{d t}{t}
$$

with

$$
\psi_{\alpha}(t)=\int_{S^{n-1}} \xi_{1}\left[t+\frac{1}{t}-2 \xi_{1}\right]^{-(n-\alpha) / 2} d \xi
$$

where $d \xi$ denotes normalized surface measure, $\xi_{1}$ is the first component of $\xi$ and $D_{\alpha}$ is specified to be the best constant in (6). Note that by monotonicity and symmetry $\psi_{\alpha}(t)$ is positive, and moreover any kernel derived from (6) will be positive-definite. Then using Young's inequality

$$
\left\|\psi_{\alpha} * h\right\|_{L^{2}\left(\mathbb{R}_{+}\right)} \leq\left\|\psi_{\alpha}\right\|_{L^{1}\left(\mathbb{R}_{+}\right)}\|h\|_{L^{2}\left(\mathbb{R}_{+}\right)}
$$

which is sharp, it suffices to calculate the $L^{1}$ norm of $\psi_{\alpha}$ to obtain the constant $D_{\alpha}$

$$
\left\|\psi_{\alpha}\right\|_{L^{1}\left(\mathbb{R}_{+}\right)}=D_{\alpha}\left[\Gamma\left(\frac{n}{2}\right) \Gamma(\alpha / 2) / 2 \pi^{\alpha} \Gamma\left(\frac{n-\alpha}{2}\right)\right] .
$$

To compute this integral, observe that

$$
\begin{aligned}
\left\|\psi_{\alpha}\right\|_{L^{1}\left(\mathbb{R}_{+}\right)} & =\int_{0}^{\infty}\left[\int_{S^{n-1}} \xi_{1}\left[t+\frac{1}{t}-2 \xi_{1}\right]^{-(n-\alpha) / 2} d \xi\right] \frac{d t}{t} \\
& =\left[\frac{2 \pi^{n / 2}}{\Gamma(n / 2)}\right]^{-1} \int_{\mathbb{R}^{n}} \frac{x \cdot y}{|x||y|} \frac{1}{|x-y|^{n-\alpha}} \frac{1}{|y|^{(n+\alpha) / 2}} d y
\end{aligned}
$$

for $|x|=1$ and $n>1$. The second integral will be calculated for the set of values $n-2>\alpha>0$. But notice that the first integral is an analytic function of the parameters $n \geq 2$ and $\beta=n-\alpha$ for some range of values. Hence any computation for some parameter domain will determine by analytic continuation the value of $\left\|\psi_{\alpha}\right\|_{L^{1}\left(\mathbb{R}_{+}\right)}$for the desired parameter interval $0<\alpha<n$ with $n>1$. Then (noting that $|x|=1$ )

$$
\begin{aligned}
& \int_{\mathbb{R}^{n}} \frac{2 x \cdot y}{|x||y|}|x-y|^{-(n-\alpha)}|y|^{-(n+\alpha) / 2} d y= \\
& \quad \int_{\mathbb{R}^{n}}|x-y|^{-(n-\alpha)}|y|^{-(n+\alpha) / 2-1} d y+\int|x-y|^{-(n-\alpha)}|y|^{-(n+\alpha) / 2+1} d y \\
& \quad-\int_{\mathbb{R}^{n}}|x-y|^{-(n-\alpha-2)}|y|^{-(n+\alpha) / 2-1} d y=I_{1}+I_{2}-I_{3}
\end{aligned}
$$


These integrals are computed by using the formula for the convolution of two Riesz potentials

$$
|x|^{-\beta} *|x|^{-\delta}=\pi^{n / 2}\left[\frac{\Gamma\left(\frac{n-\beta}{2}\right) \Gamma\left(\frac{n-\delta}{2}\right) \Gamma\left(\frac{\beta+\delta-n}{2}\right)}{\Gamma\left(\frac{\beta}{2}\right) \Gamma\left(\frac{\delta}{2}\right) \Gamma\left(\frac{2 n-\beta-\delta}{2}\right)}\right]|x|^{-(\beta+\delta-n)}
$$

with $0<\beta<n, 0<\delta<n$ and $n<\beta+\delta<2 n$. Then

$$
\begin{aligned}
& I_{1}=\pi^{n / 2}\left[\frac{\Gamma\left(\frac{\alpha}{2}\right) \Gamma\left(\frac{n-\alpha-2}{4}\right) \Gamma\left(\frac{n-\alpha+2}{4}\right)}{\Gamma\left(\frac{n-\alpha}{2}\right) \Gamma\left(\frac{n+\alpha+2}{4}\right) \Gamma\left(\frac{n+\alpha-2}{4}\right)}\right] \\
& I_{2}=\pi^{n / 2}\left[\frac{\Gamma\left(\frac{\alpha}{2}\right) \Gamma\left(\frac{n-\alpha+2}{4}\right) \Gamma\left(\frac{n-\alpha-2}{4}\right)}{\Gamma\left(\frac{n-\alpha}{2}\right) \Gamma\left(\frac{n+\alpha-2}{4}\right) \Gamma\left(\frac{n+\alpha+2}{4}\right)}\right] \\
& I_{3}=\pi^{n / 2}\left[\frac{\Gamma\left(\frac{\alpha+2}{2}\right) \Gamma\left(\frac{n-\alpha-2}{4}\right) \Gamma\left(\frac{n-\alpha-2}{4}\right)}{\Gamma\left(\frac{n-\alpha-2}{2}\right) \Gamma\left(\frac{n+\alpha+2}{4}\right) \Gamma\left(\frac{n+\alpha+2}{4}\right)}\right]
\end{aligned}
$$

and

$$
\begin{aligned}
I_{1}+I_{2}-I_{3} & =\pi^{n / 2} \frac{\Gamma\left(\frac{\alpha}{2}\right)}{\Gamma\left(\frac{n-\alpha}{2}\right)}\left[\frac{\Gamma\left(\frac{n-\alpha-2}{4}\right)}{\Gamma\left(\frac{n+\alpha+2}{4}\right)}\right]^{2}\left[2\left(\frac{n-\alpha-2}{4}\right)\left(\frac{n+\alpha-2}{4}\right)-\frac{\alpha}{2}\left(\frac{n-\alpha-2}{2}\right)\right] \\
& =\pi^{n / 2} \frac{\Gamma\left(\frac{\alpha}{2}\right)}{\Gamma\left(\frac{n-\alpha}{2}\right)}\left[\frac{\Gamma\left(\frac{n-\alpha-2}{4}\right)}{\Gamma\left(\frac{n+\alpha+2}{4}\right)}\right]^{2}\left[2\left(\frac{n-\alpha-2}{4}\right)^{2}\right]=2 \pi^{n / 2} \frac{\Gamma\left(\frac{\alpha}{2}\right)}{\Gamma\left(\frac{n-\alpha}{2}\right)}\left[\frac{\Gamma\left(\frac{n-\alpha+2}{4}\right)}{\Gamma\left(\frac{n+\alpha+2}{4}\right)}\right]^{2}
\end{aligned}
$$

This demonstrates that

$$
\left\|\psi_{\alpha}\right\|_{L^{1}\left(\mathbb{R}_{+}\right)}=\frac{\Gamma\left(\frac{n}{2}\right) \Gamma\left(\frac{\alpha}{2}\right)}{2 \Gamma\left(\frac{n-\alpha}{2}\right)}\left[\frac{\Gamma\left(\frac{n-\alpha+2}{4}\right)}{\Gamma\left(\frac{n+\alpha+2}{4}\right)}\right]^{2}
$$

and that for radial functions in (6)

$$
D_{\alpha}=\pi^{\alpha}\left[\frac{\Gamma\left(\frac{n-\alpha+2}{4}\right)}{\Gamma\left(\frac{n+\alpha+2}{4}\right)}\right]^{2} .
$$

Step 2. The Hecke-Bochner representation for $L^{2}\left(\mathbb{R}^{n}\right)$ is used to reduce the study of inequality (6)

$$
\int_{\mathbb{R}^{n}}|\nabla f|^{2}|x|^{-\alpha} d x \leq 4 \pi^{2} D_{\alpha} \int_{\mathbb{R}^{n}}|\widehat{f}(y)|^{2}|y|^{\alpha+2} d y
$$

to estimates for radial functions. For $f \in \mathcal{S}\left(\mathbb{R}^{n}\right)$

$$
f(x)=\sum_{k=0}^{\infty} f_{k}(|x|) P_{k}(x)
$$

where $P_{k}$ is a harmonic polynomial of degree $k$,

$$
P_{k}(x)=|x|^{k} Y_{k}(\xi), \quad \xi=\frac{x}{|x|}, \quad \int_{S^{n-1}}\left|Y_{k}(\xi)\right|^{2} d \xi=\frac{\omega_{n-1+2 k}}{\omega_{n-1}}
$$

$Y_{k}$ is a spherical harmonic of degree $k, \omega_{m}=$ surface area of the unit sphere $S^{m}$, and $d \xi$ is normalized surface measure on $S^{n-1}$. Then

$$
\int_{\mathbb{R}^{n}}|f|^{2} d x=\sum_{k=0}^{\infty}\left|f_{k}(|x|)\right|^{2} d x
$$

Let $\mathcal{F}_{n}$ denote the Fourier transform on $\mathbb{R}^{n}$. Bochner's relation for spherical harmonics is

$$
\mathcal{F}_{n}\left(f_{k}(|x|) P_{k}(x)\right)=i^{k} \mathcal{F}_{n+2 k}\left(f_{k}(|x|)\right) P_{k} .
$$

and the integral on the right-hand side of (6) becomes

$$
\int_{\mathbb{R}^{n}}|\widehat{f}(y)|^{2}|y|^{\alpha+2} d y=\sum_{k=0}^{\infty} \int_{\mathbb{R}^{n+2 k}}\left|\widehat{f}_{k}(|y|)\right|^{2}|y|^{\alpha+2} d y .
$$


For the integral on the left-hand side in (6)

$$
\begin{aligned}
\int_{\mathbb{R}^{n}}|\nabla f|^{2}|x|^{-\alpha} d x & =\sum_{k=0}^{\infty} \int_{\mathbb{R}^{n}}\left|\nabla\left[f_{k}(|x|) P_{k}(x)\right]\right|^{2}|x|^{-\alpha} d x \\
& =\sum_{k=0}^{\infty} \int_{\mathbb{R}^{n+2 k}}\left|\nabla f_{k}(|x|)\right|^{2}|x|^{-\alpha} d x+\sum_{k=1}^{\infty} k \alpha \int_{\mathbb{R}^{n+2 k}}\left|f_{k}(|x|)\right|^{2}|x|^{-\alpha-2} d x .
\end{aligned}
$$

Using inequality (6) for radial functions from Step 1,

$$
\int_{\mathbb{R}^{n+2 k}}\left|\nabla f_{k}(|x|)\right|^{2}|x|^{-\alpha} d x \leq 4 \pi^{2+\alpha}\left[\frac{\Gamma\left(\frac{n+2 k-\alpha+2}{4}\right)}{\Gamma\left(\frac{n+2 k+\alpha+2}{4}\right)}\right]^{2} \int_{\mathbb{R}^{n+2 k}}\left|\widehat{f}_{k}(y)\right|^{2}|y|^{\alpha+2} d y
$$

and using inequality (3)

$$
\int_{\mathbb{R}^{n+2 k}}\left|f_{k}(|x|)\right|^{2}|x|^{-\alpha-2} d x \leq \pi^{2+\alpha}\left[\frac{\Gamma\left(\frac{n+2 k-\alpha-2}{4}\right)}{\Gamma\left(\frac{n+2 k+\alpha+2}{4}\right)}\right]^{2} \int_{\mathbb{R}^{n+2 k}}\left|\widehat{f}_{k}(y)\right|^{2}|y|^{\alpha+2} d y
$$

one obtains

$$
\begin{aligned}
& \int_{\mathbb{R}^{n}}|\nabla f|^{2}|x|^{-\alpha} d x \\
& \quad \leq 4 \pi^{2+\alpha} \sum_{k=0}^{\infty}\left[\left[\frac{\Gamma\left(\frac{n+2 k-\alpha+2}{4}\right)}{\Gamma\left(\frac{n+2 k+\alpha+2}{4}\right)}\right]^{2}+\frac{k \alpha}{4}\left[\frac{\Gamma\left(\frac{n+2 k-\alpha-2}{4}\right)}{\Gamma\left(\frac{n+2 k+\alpha+2}{4}\right)}\right]^{2}\right] \int_{\mathbb{R}^{n+2 k}}\left|\widehat{f}_{k}(y)\right|^{2}|y|^{\alpha+2} d y \\
& \quad \leq 4 \pi^{2+\alpha} \max _{k}\left\{\left[\frac{\Gamma\left(\frac{n+2 k-\alpha+2}{4}\right)}{\Gamma\left(\frac{n+2 k+\alpha+2}{4}\right)}\right]^{2}\left[1+\frac{4 k \alpha}{(n+2 k-\alpha-2)^{2}}\right]\right\} \int_{\mathbb{R}^{n}}|\widehat{f}(y)|^{2}|y|^{\alpha+2} d y
\end{aligned}
$$

which demonstrates inequality (6), Theorem 4 and the equivalent Theorem 5 .

Corollary 1. For $\alpha=2$

$$
\frac{D_{2}}{\pi^{2}}= \begin{cases}\frac{144}{25}, & \text { if } n=3(k=1 \text { term }) \\ \frac{4}{3}, & \text { if } n=4(k=1 \text { term }) \\ \frac{16}{n^{2}}, & \text { if } n>4(k=0 \text { term })\end{cases}
$$

This result recovers a "classical Hardy-Rellich inequality" for $n>4$ (see remarks in [14])

$$
\int_{\mathbb{R}^{n}}|\nabla f|^{2}|x|^{-2} d x \leq \frac{4}{n^{2}} \int_{\mathbb{R}^{n}}|\Delta f|^{2} d x .
$$

Notice that for $n=8$ this inequality is entirely elementary since integrating by parts

$$
\int|\nabla f|^{2}|x|^{-2} d x=-\int f(\Delta f)|x|^{-2} d x-8 \int|f|^{2}|x|^{-4} d x \leq \frac{1}{16} \int|\Delta f|^{2} d x .
$$

In comparing terms to evaluate constants explicitly, the following fact is useful: for $0<x<y$, the ratio $\Gamma(x+\beta) / \Gamma(y+\beta)$ is decreasing for $\beta>0$. Set $F(\beta)=\ln \Gamma(x+\beta)-\ln \Gamma(y+\beta)$; then

$$
F^{\prime}(\beta)=\psi(x+\beta)-\psi(y+\beta)=-(y-x) \sum_{k=0}^{\infty}(x+\beta+k)^{-1}(y+\beta+k)^{-1}<0 .
$$

For $n=2$, the expression

$$
\left[\frac{\Gamma\left(\frac{n+2 k-\alpha+2}{4}\right)}{\Gamma\left(\frac{n+2 k+\alpha+2}{4}\right)}\right]^{2}\left(1+\frac{4 k \alpha}{(n+2 k-\alpha-2)^{2}}\right)
$$

is decreasing for $k \geq 1$ since both terms in the product are decreasing so the value of $D_{\alpha}$ is found by comparing the terms for $k=0$ and $k=1$. 
Corollary 2. For $n=2$

$$
D_{\alpha}=\pi^{\alpha}\left[\frac{\Gamma\left(\frac{3}{2}-\frac{\alpha}{4}\right)}{\Gamma\left(\frac{3}{2}+\frac{\alpha}{4}\right)}\right]^{2}\left(\frac{4+\alpha^{2}}{(2-\alpha)^{2}}\right) .
$$

Proof. Set $\beta=\alpha / 4$ with $0 \leq \beta<\frac{1}{2}$ since $\alpha<n=2$. Then to see that the $k=1$ term is larger than the $k=0$ term, consider the log of the ratio of these two terms and it suffices to show that

$$
F(\beta)=\ln \Gamma\left(\frac{1}{2}-\beta\right)+\ln \Gamma(1+\beta)-\ln \Gamma(1-\beta)-\ln \Gamma\left(\frac{3}{2}+\beta\right)+\frac{1}{2} \ln \left(\frac{1}{4}+\beta^{2}\right)
$$

is positive for $\beta>0$ which will follow by showing that $F(\beta)$ is increasing with $f(0)=0$.

$$
\begin{aligned}
F^{\prime}(\beta)= & -4\left(\frac{1}{2}-\beta\right)+\psi(1+\beta)+\psi(1-\beta)-\psi\left(\frac{3}{2}+\beta\right)+\frac{4 \beta}{1+4 \beta^{2}} \\
= & \left(\frac{1}{2}+2 \beta\right) \sum_{k=0}^{\infty}\left[\left(\frac{1}{2}-\beta+k\right)^{-1}(1+\beta+k)^{-1}-(1-\beta+k)^{-1}\left(\frac{3}{2}+\beta+k\right)^{-1}\right] \\
& +\frac{4 \beta}{1+4 \beta^{2}}>0
\end{aligned}
$$

$F^{\prime}(\beta)>0$ and $F(0)=0$ ensure that $F(\beta)$ is positive.

Corollary 3. For $n \geq 3$

$$
D_{\alpha}=\pi^{\alpha} \max _{k=0,1}\left\{\left[\frac{\Gamma\left(\frac{n+2 k-\alpha+2}{4}\right)}{\Gamma\left(\frac{n+2 k+\alpha+2}{4}\right)}\right]^{2}\left(1+\frac{4 k \alpha}{(n+2 k-\alpha-2)^{2}}\right)\right\} .
$$

Proof. The objective here is to show that only the $k=0$ and $k=1$ terms in Theorem 4 need to be compared. That is, the only functions that are necessary to consider in Theorem 4 are those contained in the span of functions with spherical harmonics up to degree one. Set $\beta=2 k$ and show that

$$
G(\beta)=\ln \left\{\left[\frac{\Gamma\left(\frac{n+\beta-\alpha+2}{4}\right)}{\Gamma\left(\frac{n+\beta+\alpha+2}{4}\right)}\right]\left(1+\frac{2 \beta \alpha}{(n+\beta-\alpha-2)^{2}}\right)^{1 / 2}\right\}
$$

is decreasing for $\beta \geq 2$ with $0<\alpha<n$; then

$$
\begin{aligned}
G^{\prime}(\beta)=\frac{1}{4}[\psi & \left.\left(\frac{n+\beta-\alpha+2}{4}\right)-\psi\left(\frac{n+\beta+\alpha+2}{4}\right)\right] \\
& +\alpha\left[1-\frac{2 \beta}{n+\beta-\alpha-2}\right]\left[(n+\beta-\alpha-2)^{2}+2 \beta \alpha\right]^{-1} \\
=\alpha & {\left[-\frac{1}{8} \sum_{k=0}^{\infty}\left[\left(\frac{n+\beta+2}{4}+k\right)^{2}-\frac{\alpha^{2}}{16}\right]^{-1}\right.} \\
& \left.+\frac{(n-\beta-\alpha-2)}{(n+\beta-\alpha-2)}\left[(n+\beta-\alpha-2)^{2}+2 \beta \alpha\right]^{-1}\right] .
\end{aligned}
$$

This derivative is clearly negative for $n=3,4$ and $\beta \geq 2, n>\alpha>0$ since $n-\beta-\alpha-2<0$. Now consider $n \geq 5$ with $0<\alpha<n$, and use a Riemann sum to approximate the first term from above:

$$
\begin{gathered}
\frac{1}{8} \sum_{k=0}^{\infty}\left[\left(\frac{n+\beta+2}{4}+k\right)^{2}-\frac{\alpha^{2}}{16}\right]^{-1}>\frac{1}{8} \sum_{k=0}^{\infty}\left(\frac{n+\beta+2}{4}+k\right)^{-2} \\
>\frac{1}{8} \int_{0}^{\infty}\left(\frac{n+\beta+2}{4}+x\right)^{-2} d x=\frac{1}{2}(n+\beta+2)^{-1} .
\end{gathered}
$$


Then

$$
G^{\prime}(\beta)<\alpha\left[-\frac{1}{2}(n+\beta+2)^{-1}+\frac{n-\beta-\alpha-2}{n+\beta-\alpha-2}\left[(n+\beta-2)^{2}+\alpha^{2}-2 \alpha(n-2)\right]^{-1}\right] .
$$

The right-hand expression is negative if

$$
\frac{n-\beta-\alpha-2}{n+\beta-\alpha-2}<\frac{(n+\beta-2)^{2}+\alpha^{2}-2 \alpha(n-2)}{2(n+\beta+2)} ;
$$

this is clearly the case if $n-\beta-\alpha-2<0$ so set $\delta=n-\beta-\alpha-2$ and consider the expression

$$
\frac{-\delta}{\delta+2 \beta}+\frac{(2 \beta+\delta+\alpha)^{2}+\alpha^{2}-2 \alpha(\delta+\beta+\alpha)}{2(\delta+2 \beta+\alpha+4)}
$$

or

$$
\begin{aligned}
H(\delta) & =(\delta+2 \beta)\left[(2 \beta+\delta+\alpha)^{2}+\alpha^{2}-2 \alpha(\delta+\beta+\alpha)\right]-2 \delta(\delta+2 \beta+\alpha+4) \\
& =(\delta+2 \beta)(\delta+2 \beta+\alpha)^{2}-(\delta+2 \beta)\left(\alpha^{2}+2 \alpha \delta+2 \alpha \beta\right)-2 \delta(\delta+2 \beta+\alpha+4) \\
& =(\delta+2 \beta)\left[\delta^{2}+4 \beta^{2}+2 \beta \alpha+(4 \beta-2 \delta]-2 \delta(\alpha+4)>0\right.
\end{aligned}
$$

for $\beta \geq 2$ and the positivity is clear for the case $\delta>0 . H(\delta)>0$ implies that $G^{\prime}(\beta)<0$, and this completes the proof of Corollary 3 .

Theorem 6 (Hardy-Rellich trace inequality). For $f \in \mathcal{S}\left(\mathbb{R}^{n}\right), n \geq 2$

$$
\begin{aligned}
& \int_{\mathbb{R}^{n}}|\nabla f|^{2}|x|^{-1} d x \leq \frac{D_{1}}{2 \pi} \int_{\mathbb{R}^{n}}\left|(-\Delta)^{3 / 4} f\right|^{2} d x \\
& \frac{D_{1}}{2 \pi}= \begin{cases}\frac{5}{2}\left[\frac{\Gamma\left(\frac{5}{4}\right)}{\Gamma\left(\frac{7}{4}\right)}\right]^{2}, & n=2(k=1 \text { term }) \\
\frac{\pi}{4}, & n=3(k=1 \text { term }) \\
\frac{1}{2}\left[\frac{\Gamma\left(\frac{n+1}{4}\right)}{\Gamma\left(\frac{n+3}{4}\right)}\right]^{2}, & n \geq 4(k=0 \text { term }) .\end{cases}
\end{aligned}
$$

Proof. Using Corollary 3, determine the maximum of the two terms $k=0,1$ in Theorem 4. From Corollary 2 for $n=2$ and by explicit calculation for $n=3$, one observes that the $k=1$ term is larger. For higher dimensions, consider the log of the ratio of the $k=1$ term to the $k=0$ term: set $w=n / 4$ and define for $w \geq 1$

$$
\Lambda(w)=\ln \left\{\left[\Gamma\left(w+\frac{3}{4}\right)^{4} / \Gamma\left(w+\frac{1}{4}\right)^{2} \Gamma\left(w+\frac{5}{4}\right)^{2}\right]\left(1+\frac{4}{(4 w-1)^{2}}\right)\right] .
$$

Observe that by Stirling's formula, $\Lambda(w) \rightarrow 0$ as $w \rightarrow \infty$ and

$$
\Lambda(1)=\ln \left[\frac{117}{25} \Gamma\left(\frac{3}{4}\right)^{4} / \Gamma\left(\frac{1}{4}\right)^{4}\right] \simeq-2.796 .
$$

Since $\Lambda(1)$ is negative, the $k=0$ term is largest for $n=4$.

$$
\begin{aligned}
\Lambda^{\prime}(w) & =4\left[\psi\left(w+\frac{3}{4}\right)-\psi\left(w+\frac{1}{4}\right)\right]+\frac{8(4 w-1)}{(4 w-1)^{2}+4}-\frac{32 w}{16 w^{2}-1} \\
& =2 \sum_{k=0}^{\infty}\left[\left(k+w+\frac{1}{2}\right)^{2}-\frac{1}{16}\right]^{-1}+\frac{8(4 w-1)}{(4 w-1)^{2}+4}-\frac{32 w}{\left(16 w^{2}-1\right)} \\
& >\frac{4}{2 w+1}+\frac{8(4 w-1)}{(4 w-1)^{2}+4}-\frac{32 w}{\left(16 w^{2}-1\right)}>0
\end{aligned}
$$


for $w \geq 1$ since

$$
\frac{4}{2 w+1}+\frac{8(4 w-1)}{(4 w-1)^{2}+4}-\frac{32 w}{16 w^{2}-1}>\frac{4}{2 w+1}+\frac{8}{4 w+1}-\frac{8}{4 w-1}>0
$$

for this range of values. Hence $\Lambda(w)$ is increasing for $w \geq 1$ or $n \geq 4$ and since the limit at infinity is zero, $\Lambda(w)$ must be negative for all $w \geq 1$ and the $k=0$ term is largest for $n \geq 4$. This completes the argument for Theorem 6 .

\section{Theorem 7.}

(A) For $n-2 \leq \alpha<n$

$$
D_{\alpha}=\pi^{\alpha}\left[\Gamma\left(\frac{n-\alpha}{4}+1\right) / \Gamma\left(\frac{n+\alpha}{4}+1\right)\right]^{2}\left(1+\frac{4 \alpha}{(n-\alpha)^{2}}\right) .
$$

(B) For $n \geq 3$ and $\alpha$ sufficiently near 0

$$
D_{\alpha}=\pi^{\alpha}\left[\Gamma\left(\frac{n-\alpha}{4}+\frac{1}{2}\right) / \Gamma\left(\frac{n+\alpha}{4}+\frac{1}{2}\right)\right]^{2} .
$$

Moreover, for $n \geq 4$ this value holds when $0<\alpha \leq n-3$.

(C) For large $n$ and fixed $\alpha, D_{\alpha} \simeq\left(\frac{4 \pi}{n}\right)^{\alpha}$.

Proof. The case $n=2$ is contained in Corollary 2. The first step to prove part (A) will be to set $\alpha=n-2$ and consider the $\log$ of the ratio of the $k=1$ term to the $k=0$ term. Then for

$$
\Lambda=\ln \left\{\left[\frac{\Gamma\left(\frac{3}{2}\right) \Gamma\left(\frac{n}{2}\right)}{\Gamma\left(\frac{n+1}{2}\right) \Gamma(1)}\right]^{2}(n-1)\right\},
$$

treat $n=w$ as a continuous variable and calculate $\Lambda^{\prime}(w)$.

$$
\begin{gathered}
\Lambda^{\prime}(w)=\frac{1}{w-1}+\psi\left(\frac{w}{2}\right)-\psi\left(\frac{w+1}{2}\right) \\
\psi\left(\frac{w+1}{2}\right)-\psi\left(\frac{w}{2}\right)=2 \int_{0}^{\infty} \frac{e^{-w t}}{1+e^{-t}} d t .
\end{gathered}
$$

This formula follows from the Gauss integral representation for $\psi$ (see Whittaker and Watson, page 247). Set $\delta=w-1$ and write for $\delta>1$

$$
\Lambda^{\prime}=\frac{1}{\delta}\left[1-2 \delta \int_{0}^{\infty} \frac{e^{-\delta t}}{1+e^{t}} d t\right]=\frac{2}{\delta} \int_{0}^{\infty} \frac{e^{t} e^{-\delta t}}{\left(1+e^{t}\right)^{2}} d t>0 .
$$

Since $\Lambda^{\prime}>0, \Lambda$ as a continuous function of $w$ increases from $-\infty$ to $\ln (\pi / 2) . \Lambda(2)=0$ then implies that $\Lambda(n)>0$ for $n \geq 3$ and verifies the claim in part (A) for $\alpha=n-2$.

The estimates obtained here for $\psi$ using both Riemann sums and the Gauss integral are expressed in the following lemma.

Lemma. For $w>1$

$$
\frac{2}{2 w+1}<\psi\left(\frac{w+1}{2}\right)-\psi\left(\frac{w}{2}\right)< \begin{cases}\frac{1}{w}+\frac{2}{w(w+1)}, & 1<w \leq 3 \\ \frac{1}{w-1}, & 3 \leq w .\end{cases}
$$

To complete the proof of part (A), set $\alpha=n-2+2 \delta$ with $0<\delta<1$ and consider

$$
\Lambda(\delta)=\ln \left\{\left[\frac{\Gamma\left[\frac{n+\delta}{2}\right] \Gamma\left[\frac{3-\delta}{2}\right]}{\Gamma\left[\frac{n+\delta+1}{2}\right] \Gamma\left[1-\frac{\delta}{2}\right]}\right]^{2}\left(\frac{n+\delta^{2}-1}{(1-\delta)^{2}}\right)\right\} .
$$


Note by using the lemma above

$$
\begin{gathered}
\Lambda^{\prime}(\delta)=\psi\left(\frac{n+\delta}{2}\right)-\psi \\
\left.+\frac{n+\delta+1}{2}\right)+\psi\left(1-\frac{\delta}{2}\right)-\psi\left(\frac{3}{2}-\frac{\delta}{2}\right)+\frac{2 \delta}{n+\delta^{2}-1}+\frac{2}{\delta-1} \\
>\frac{2 \delta}{n+\delta^{2}-1}+\frac{1}{1-\delta}-\frac{1}{n+\delta-1}>0
\end{gathered}
$$

so $\Lambda(\delta)$ is increasing for $0<\delta<1$ and since $\Lambda(0)>0 \quad \Lambda(\delta)$ is positive and the result in part (A) is verified.

The purpose of parts (A) and (B) is to demonstrate that for dimension at least four or larger there are definite ranges of the parameter $\alpha$ where either the $k=0$ or $k=1$ terms give the precise constant for Theorem 4 For $n=2$ the $k=1$ term is largest for all $\alpha$. For $n=3$, the $k=1$ term is largest except for a small neighborhood of $\alpha=0$. For $n \geq 4$ one can identify a definite interval in the parameter range for $\alpha$ where the transition between the two terms for determining the maximum value for $D_{\alpha}$ occurs. Consider the $\log$ of the ratio of the two terms

$$
\Lambda=\ln \left\{\left[\frac{\Gamma\left(\frac{n-\alpha}{4}+1\right)}{\Gamma\left(\frac{n+\alpha}{4}+1\right)} \frac{\Gamma\left(\frac{n+\alpha}{4}+\frac{1}{2}\right)}{\Gamma\left(\frac{n-\alpha}{4}+\frac{1}{2}\right)}\right]^{2}\left(1+\frac{4 \alpha}{(n-\alpha)^{2}}\right)\right\}
$$

which for $n=3$ becomes

$$
\Lambda=\ln \left\{\left[\frac{\Gamma\left(\frac{7-\alpha}{4}\right) \Gamma\left(\frac{5+\alpha}{4}\right)}{\Gamma\left(\frac{7+\alpha}{4}\right) \Gamma\left(\frac{5-\alpha}{4}\right)}\right]^{2}\left(\frac{\alpha^{2}-2 \alpha+9}{(3-\alpha)^{2}}\right)\right\} .
$$

For small values of $\alpha, \Lambda$ is still positive which means that the $k=1$ term is largest, e.g. for $\alpha=0.2$, $\Lambda \simeq 0.0021145$, but for $\alpha=0,1, \Lambda \simeq-0.00103461$. Note

$$
\Lambda^{\prime}(0)=-\psi(7 / 4)+\psi(5 / 4)+4 / 9 \simeq-0.0304815
$$

which requires since $\Lambda(0)=0$ that near zero, $\Lambda(\alpha)<0$ and the $k=0$ term is largest.

To show the case $n \geq 4$ for part (B), set $n=\alpha+2 \delta$ with $\delta \geq 3 / 2$; then

$$
\Lambda=\ln \left\{\left[\frac{\Gamma\left(\frac{\delta}{2}+1\right) \Gamma\left(\frac{\alpha+\delta}{2}+\frac{1}{2}\right)}{\Gamma\left(\frac{\delta}{2}+\frac{1}{2}\right) \Gamma\left(\frac{\alpha+\delta}{2}+1\right)}\right]^{2}\left(1+\frac{\alpha}{\delta^{2}}\right)\right\}
$$

which can now be viewed as a function of $\alpha$ for $0<\alpha \leq n-2 \delta$ and $\delta \geq 3 / 2$. For this range of values of $\delta$

$$
\begin{aligned}
\Lambda^{\prime}(\alpha) & =\psi\left(\frac{\alpha+\delta+1}{2}\right)-\psi\left(\frac{\alpha+\delta}{2}+1\right)+\frac{1}{\alpha+\delta^{2}} \\
& <-\frac{2}{\alpha+\delta+2}+\frac{1}{\alpha+\delta^{2}}<0 .
\end{aligned}
$$

Since $\Lambda(0)=0$, the desired value of $\Lambda$ for $\alpha=n-2 \delta$ must be negative which will imply that the $k=0$ term is largest for $0<\alpha \leq n-3$ and $n \geq 4$. This completes the proof of part (B).

Part (C) follows directly as an application of Stirling's formula

$$
\Gamma(z+a) \simeq \sqrt{2 \pi} e^{-z} z^{z+a-\frac{1}{2}} \text { as } z \rightarrow \infty
$$

since for fixed $\alpha$ and large $n$, the value $D_{\alpha}$ will use the $k=0$ term

$$
D_{\alpha} \simeq\left(\frac{4 \pi}{n}\right)^{\alpha} \text {. }
$$

Note that for $\alpha=2$, this becomes an exact relation for $n \geq 5$ : $D_{2}=(4 \pi / n)^{2}$. 


\section{LOGARITHMiC UNCERTAINTY}

The basic relation for Pitt's inequality with gradient terms

$$
\int_{\mathbb{R}^{n}}|\nabla f|^{2}|x|^{-\alpha} d x \leq 4 \pi^{2} D_{\alpha} \int_{\mathbb{R}^{n}}|\widehat{f}(y)|^{2}|y|^{\alpha+2} d y
$$

becomes an equality at $\alpha=0\left(D_{0}=1\right)$ so it can be differentiated at this value of $\alpha$. Corollary 2 and Theorem 7 express the value of $D_{\alpha}$ needed for this calculation.

Theorem 8. For $f \in \mathcal{S}\left(\mathbb{R}^{n}\right)$ and $n \geq 2$

$$
\begin{gathered}
\int_{\mathbb{R}^{n}} \ln |x||\nabla f|^{2} d x+4 \pi^{2} \int_{\mathbb{R}^{n}} \ln |y||y|^{2}|\widehat{f}(y)|^{2} d y \geq E \int_{\mathbb{R}^{n}}|\nabla f|^{2} d x \\
E= \begin{cases}\psi\left(\frac{3}{2}\right)-\ln \pi-1, & n=2 \\
\psi\left(\frac{n}{4}+\frac{1}{2}\right)-\ln \pi, & n \geq 3 .\end{cases}
\end{gathered}
$$

The increase in the constant here over the corresponding value in Theorem 2 reflects the comparison of the integrals

$$
\int_{\mathbb{R}^{n}} \ln |x||\nabla f|^{2} d x \geq \int_{\mathbb{R}^{n}} \ln |x|\left|\mathcal{F}^{-1}(2 \pi|y| \widehat{f}(y))\right|^{2} d x .
$$

\section{Iterated Stein-Weiss potentials}

The Stein-Weiss potentials discussed here act at the spectral level, that is, in terms of $L^{2}$ estimates. In the context of using these potentials to define a linear operator on $L^{2}\left(\mathbb{R}^{n}\right)$, it is natural to examine iterated applications. For example, the Stein-Weiss potential with $0<\alpha<n$

$$
\int_{\mathbb{R}^{n} \times \mathbb{R}^{n}} f(x)|x|^{-\alpha / 2}|x-y|^{-(n-\alpha)}|y|^{-\alpha / 2} f(y) d x d y
$$

corresponds to the linear operator

$$
g \longrightarrow|x|^{-\alpha / 2}\left(|x|^{-\left(n-\frac{\alpha}{2}\right)} * g\right)
$$

and inequality (5) can be rephrased

$$
\left\||x|^{-\alpha / 2}\left(|x|^{-\left(n-\frac{\alpha}{2}\right)} * g\right)\right\|_{L^{2}\left(\mathbb{R}^{n}\right)} \leq \pi^{n / 2}\left[\frac{\Gamma\left(\frac{\alpha}{4}\right) \Gamma\left(\frac{n-\alpha}{4}\right)}{\Gamma\left(\frac{n}{2}-\frac{\alpha}{4}\right) \Gamma\left(\frac{n+\alpha}{4}\right)}\right]\|g\|_{L^{2}\left(\mathbb{R}^{n}\right)}
$$

or as a weighted Sobolev inequality

$$
\|h\|_{L^{2}\left(\mathbb{R}^{n}\right.} \leq 2^{-\alpha / 2}\left[\frac{\Gamma\left(\frac{n-\alpha}{4}\right)}{\Gamma\left(\frac{n+\alpha}{4}\right)}\right]\left\|(-\Delta)^{\alpha / 4}\left(|x|^{\alpha / 2} h\right)\right\|_{L^{2}\left(\mathbb{R}^{n}\right)}
$$

which further implies by using the $\left\|T^{*} T\right\|=\|T\|^{2}$ argument that

$$
\|h\|_{L^{2}\left(\mathbb{R}^{n}\right.} \leq 2^{-\alpha}\left[\frac{\Gamma\left(\frac{n-\alpha}{4}\right)}{\Gamma\left(\frac{n+\alpha}{4}\right)}\right]^{2}\left\||x|^{\alpha / 2}(-\Delta)^{\alpha / 2}\left(|x|^{\alpha / 2} h\right)\right\|_{L^{2}\left(\mathbb{R}^{n}\right)}
$$

These latter inequalities extend to include successive applications of powers of $|x|$ and $(-\Delta)^{1 / 2}$ and correspond to iterated Stein-Weiss potentials subject to growth constraints on the size of the powers and the dilation constraint that the sum of the powers of $|x|$ must equal the sum of the powers of $(-\Delta)^{1 / 2}$. At the second iteration level, this algorithm leads to a result that includes the Maz'ya-Eilertsen inequality:

$$
\|h\|_{L^{2}\left(\mathbb{R}^{n}\right)} \leq C\left\|(-\Delta)^{\rho / 4}|x|^{\sigma / 2}(-\Delta)^{\beta / 4}\left(|x|^{\alpha / 2} h\right)\right\|_{L^{2}\left(\mathbb{R}^{n}\right)}
$$

\footnotetext{
${ }^{1}$ This inequality corresponds to the case $\mu=\lambda$ for inequality (7) in [7.
} 
with $\sigma+\alpha=\rho+\beta$, and

$$
\begin{aligned}
& \int_{\left(\mathbb{R}^{n}\right)^{4}} g(w)|w|^{-\rho / 2}|x-w|^{-(n-\sigma / 2)}|x|^{-\beta / 2}|x-y|^{-(n-\alpha)}|y|^{-\beta / 2}|y-v|^{-(n-\sigma / 2)}|v|^{-\rho / 2} \\
& g(v) d x d y d w d v \leq C_{1} \int_{\mathbb{R}^{n}}|g|^{2} d x .^{2}
\end{aligned}
$$

By applying symmetrization to inequality (23), one reduces the calculation of a sharp constant to considering non-negative radial decreasing functions. Since $g$ can now be taken to be radial, set $|w|=p$ $|x|=t,|y|=s,|v|=r$ with $u(p)=|w|^{n / 2} g(w)$, and inequality (23) is reduced to a convolution inequality on the multiplicative group $\mathbb{R}_{+}$

$$
\|\varphi * u\|_{L^{2}\left(\mathbb{R}_{+}\right)} \leq\|\varphi\|_{L^{1}\left(\mathbb{R}_{+}\right)}\|u\|_{L^{2}\left(\mathbb{R}_{+}\right)}
$$

where $\varphi=\kappa * \psi_{\alpha} * \kappa$ with

$$
\psi_{\alpha}(t)=\int_{S^{n-1}}\left[t+\frac{1}{t}-2 \xi_{1}\right]^{-(n-\alpha) / 2} d \xi, \quad \kappa(t)=t^{-\rho / 2+\sigma / 4} \psi_{\sigma / 2}(t)
$$

where $d \xi=$ normalized surface measure on $S^{n-1}$.

$$
\begin{gathered}
\|\varphi\|_{L^{1}\left(\mathbb{R}_{+}\right)}=\left\|\kappa * \psi_{\alpha} * \kappa\right\|_{L^{1}\left(\mathbb{R}_{+}\right)}=\left(\|\kappa\|_{L^{1}\left(\mathbb{R}_{+}\right)}\right)^{2}\left\|\psi_{\alpha}\right\|_{L^{1}\left(\mathbb{R}_{+}\right)} \\
\left\|\psi_{\alpha}\right\|_{L^{1}\left(\mathbb{R}_{+}\right)}=\frac{\Gamma\left(\frac{n}{2}\right) \Gamma\left(\frac{\alpha}{2}\right)}{2 \Gamma\left(\frac{n-\alpha}{2}\right)}\left[\frac{\Gamma\left(\frac{n-\alpha}{4}\right)}{\Gamma\left(\frac{n+\alpha}{4}\right)}\right]^{2}, \quad 0<\alpha<n \\
\|\kappa\|_{L^{1}\left(\mathbb{R}_{+}\right)}=\int_{0}^{\infty} t^{-\rho / 2+\sigma / 4}\left[\int_{S^{n-1}}\left[t+\frac{1}{t}-2 \xi_{1}\right]^{-\left(n-\frac{\sigma}{2}\right) / 2} d \xi\right] \frac{d t}{t} \\
=\left[\frac{2 \pi^{n / 2}}{\Gamma(n / 2)}\right]^{-1} \int_{\mathbb{R}^{n}}|x-y|^{-\left(n-\frac{\sigma}{2}\right)}|y|^{-(\rho+n) / 2} d y, \quad|x|=1 \\
=\frac{\Gamma\left(\frac{n}{2}\right) \Gamma\left(\frac{\sigma}{4}\right) \Gamma\left(\frac{n-\rho}{2}\right) \Gamma\left[\frac{n+\rho-\sigma}{4}\right]}{2 \Gamma\left(\frac{n}{2}-\frac{\sigma}{4}\right) \Gamma\left(\frac{\rho+n}{2}\right) \Gamma\left[\frac{n+\sigma-\rho}{4}\right]} \\
C_{1}=\left[\frac{2 \pi^{n / 2}}{\Gamma(n / 2)}\right]^{3}\|\varphi\|_{L^{1}\left(\mathbb{R}_{+}\right)} \\
=\pi^{3 n / 2} \frac{\Gamma\left(\frac{\alpha}{2}\right)}{\Gamma\left(\frac{n-\alpha}{2}\right)}\left[\frac{\Gamma\left(\frac{n-\alpha}{4}\right)}{\Gamma\left(\frac{n+\alpha}{4}\right)} \frac{\Gamma\left(\frac{\sigma}{4}\right)}{\Gamma\left(\frac{n}{2}-\frac{\sigma}{4}\right)} \frac{\Gamma\left(\frac{n-\rho}{2}\right)}{\Gamma\left(\frac{n+\rho}{2}\right)} \frac{\Gamma\left(\frac{n+\rho-\sigma}{4}\right)}{\Gamma\left(\frac{n+\sigma-\rho}{4}\right)}\right]^{2}
\end{gathered}
$$

and

$$
C=2^{-(\alpha+\sigma) / 2}\left[\frac{\Gamma\left(\frac{n-\alpha}{4}\right)}{\Gamma\left(\frac{n+\alpha}{4}\right)} \frac{\Gamma\left(\frac{n-\rho}{2}\right)}{\Gamma\left(\frac{n+\rho}{2}\right)} \frac{\Gamma\left(\frac{n+\rho-\sigma}{4}\right)}{\Gamma\left(\frac{n+\sigma-\rho}{4}\right)}\right] .
$$

These calculations comprise the proof of the following theorem:

\footnotetext{
${ }^{2}$ The case $\rho=0, \sigma=\mu, \beta=2 \lambda$ and $\alpha=2 \lambda-\mu$ corresponds to inequality (7) in [7].
} 
Theorem 9. For $g, h \in \mathcal{S}\left(\mathbb{R}^{n}\right)$ and $0<\alpha, \beta, \rho, \sigma<n, \alpha+\sigma=\beta+\rho$

$$
\begin{gathered}
\int_{\left(\mathbb{R}^{n}\right)^{4}} g(w)|w|^{-\frac{\rho}{2}}|x-w|^{-\left(n-\frac{\sigma}{2}\right)}|x|^{-\frac{\beta}{2}}|x-y|^{-(n-\alpha)}|y|^{-\frac{\beta}{2}}|y-v|^{-\left(n-\frac{\sigma}{2}\right)}|v|^{-\frac{\rho}{2}} g(v) d x d y d w d v \\
\leq B_{\alpha, \rho, \sigma} \int_{\mathbb{R}^{n}}|g|^{2} d x \\
B_{\alpha, \rho, \sigma}=\pi^{3 n / 2} \frac{\Gamma\left(\frac{\alpha}{2}\right)}{\Gamma\left(\frac{n-\alpha}{2}\right)}\left[\frac{\Gamma\left(\frac{n-\alpha}{4}\right)}{\Gamma\left(\frac{n+\alpha}{4}\right)} \frac{\Gamma\left(\frac{\sigma}{4}\right)}{\Gamma\left(\frac{n}{2}-\frac{\sigma}{4}\right)} \frac{\Gamma\left(\frac{n-\rho}{2}\right)}{\Gamma\left(\frac{n+\rho}{2}\right)} \frac{\Gamma\left(\frac{n+\rho-\sigma}{4}\right)}{\Gamma\left(\frac{n+\sigma-\rho}{4}\right)}\right]^{2} \\
\|h\|_{L^{2}\left(\mathbb{R}^{n}\right)} \leq C_{\alpha, \rho, \sigma}\left\|(-\Delta)^{\rho / 4}|x|^{\sigma / 2}(-\Delta)^{\beta / 4}\left(|x|^{\alpha / 2} h\right)\right\|_{L^{2}\left(\mathbb{R}^{n}\right)} \\
C_{\alpha, \rho, \sigma}=2^{-(\alpha+\sigma) / 2}\left[\frac{\Gamma\left(\frac{n-\alpha}{4}\right)}{\Gamma\left(\frac{n+\alpha}{4}\right)} \frac{\Gamma\left(\frac{n-\rho}{2}\right)}{\Gamma\left(\frac{n+\rho}{2}\right)} \frac{\Gamma\left(\frac{n+\rho-\sigma}{4}\right)}{\Gamma\left(\frac{n+\sigma-\rho}{4}\right)}\right] .
\end{gathered}
$$

Remark. The papers 7 and [16 consider a broader set of problems which allow parameters that lie outside the normal range of values for fractional integrals.

\section{Pitt's inequality With iterated GRadients}

Pitt's inequality with gradient terms

$$
\int_{\mathbb{R}^{n}} \Phi(1 /|x|)|\nabla f|^{2} d x \leq 4 \pi^{2} D_{\Phi} \int_{\mathbb{R}^{n}} \Phi(|y|)|y|^{2}|\widehat{f}(y)|^{2} d y
$$

is an intrinsic refinement of the classical inequality and extends naturally to iterated gradients

$$
\int_{\mathbb{R}^{n}} \Phi(1 /|x|)\left|\nabla^{\ell} f\right|^{2} d x \leq\left(4 \pi^{2}\right)^{\ell} D_{\Phi, \ell} \int_{\mathbb{R}^{n}} \Phi(y)|y|^{2 \ell}|\widehat{f}(y)|^{2} d y
$$

where

$$
\left|\nabla^{\ell} f\right|^{2}=\sum_{p_{1}=1}^{n} \cdots \sum_{p_{\ell}=1}^{n}\left(\frac{\partial}{\partial x_{p_{1}}} \cdots \frac{\partial}{\partial x_{p_{\ell}}} f\right)^{2}
$$

and $D_{\Phi, \ell}<C_{\Phi}$ (see equation (10).

Theorem 10. For $f \in \mathcal{S}\left(\mathbb{R}^{n}\right)$ and $0<\alpha<n, n>1$

$$
\int_{\mathbb{R}^{n}}\left|\nabla^{\ell} f\right|^{2}|x|^{-\alpha} d x \leq\left(4 \pi^{2}\right)^{\ell} D_{\alpha, \ell} \int_{\mathbb{R}^{n}}|\widehat{f}(y)|^{2}|y|^{\alpha+2 \ell} d y .
$$

For $f \in L^{2}\left(\mathbb{R}^{n}\right)$

$$
\begin{gathered}
\left|\int_{\mathbb{R}^{n} \times \mathbb{R}^{n}} f(x) \frac{1}{|x|^{\alpha / 2}}\left(\frac{x \cdot y}{|x||y|}\right)^{\ell} \frac{1}{|x-y|^{n-\alpha}} \frac{1}{|y|^{\alpha / 2}} f(y) d x d y\right| \\
\leq\left[\pi^{\frac{n}{2}-\alpha} \Gamma\left(\frac{\alpha}{2}\right) / \Gamma\left(\frac{n-\alpha}{2}\right)\right] D_{\alpha, \ell} \int_{\mathbb{R}^{n}}|f|^{2} d x .
\end{gathered}
$$

Observe that when $\ell$ is even, the kernel in (28) is positive and the issue of calculating $D_{\alpha, \ell}$ is reduced to considering radial functions.

Lemma. For $F, G \in L^{2}\left(S^{n}\right)$ and $K(\xi \cdot \eta) \geq 0$ with $\xi, \eta \in S^{n}$

$$
\left|\int_{S^{n} \times S^{n}} F(\xi) K(\xi \cdot \eta) G(\eta) d \xi d \eta\right| \leq\left(\int_{S^{n}} K\left(\xi_{1}\right) d \xi\right)\|F\|_{L^{2}\left(S^{n}\right)}\|G\|_{L^{2}\left(S^{n}\right)}
$$


Proof. Split the integrand into the product of two parts, $F \sqrt{K}$ and $G \sqrt{K}$, and apply Hölder's inequality.

For radial functions, inequality (28) is equivalent to the convolution inequality on the group $\mathbb{R}_{+}$

$$
\begin{gathered}
\left|\int_{\mathbb{R}_{+} \times \mathbb{R}_{+}} h(t) \psi_{\alpha, \ell}(s / t) h(s) \frac{d s}{s} \frac{d t}{t}\right| \leq D_{\alpha, \ell}\left[\frac{\Gamma\left(\frac{n}{2}\right) \Gamma\left(\frac{\alpha}{2}\right)}{2 \pi^{\alpha} \Gamma\left(\frac{n-\alpha}{2}\right)}\right] \int_{\mathbb{R}_{+}}|h|^{2} \frac{d t}{t} \\
\psi_{\alpha, \ell}(t)=\int_{S^{n-1}}\left(\xi_{1}\right)^{\ell}\left[t+\frac{1}{5}-2 \xi_{1}\right]^{-(n-\alpha) / 2} d \xi
\end{gathered}
$$

Hence, for $\ell$ even

$$
D_{\alpha, \ell}=\frac{2 \pi^{\alpha} \Gamma\left(\frac{n-\alpha}{2}\right)}{\Gamma\left(\frac{n}{2}\right) \Gamma\left(\frac{\alpha}{2}\right)}\left\|\psi_{\alpha, \ell}\right\|_{L^{1}\left(\mathbb{R}_{+}\right)} .
$$

For $\ell=2$, observe that

$$
\psi_{\alpha, n, 2}=\psi_{\alpha, n, 0}-\left(\frac{n-1}{n}\right) \psi_{\alpha+2, n+2,0}
$$

and that from equation (3)

$$
D_{\alpha, 0}=\pi^{\alpha}\left[\frac{\Gamma\left(\frac{n-\alpha}{4}\right)}{\Gamma\left(\frac{n+\alpha}{4}\right)}\right]^{2}
$$

which then provides: $D_{\alpha, n, 2}=D_{\alpha, n, 0}-\frac{\alpha}{4}(n-1) D_{\alpha+2, n+2,0}$

\section{Corollary.}

$$
D_{\alpha, 2}=\pi^{\alpha}\left[\frac{\Gamma\left(\frac{n-\alpha}{4}\right)}{\Gamma\left(\frac{n+\alpha}{4}\right)}\right]^{2}\left[\frac{(n-\alpha)^{2}+4 \alpha}{(n+\alpha)^{2}}\right] .
$$

More generally, this argument determines a recursion formula for computing constants for the case of radial functions which includes the case when $\ell$ is an even integer:

$$
D_{\alpha, n, \ell+2}=D_{\alpha, n, \ell}-\frac{\alpha}{4}(n-1) D_{\alpha+2, n+2, \ell}
$$

\section{ACKNOWLEDGEMENTS}

The computer program mathematica was used to aid some numerical calculations.

\section{REFERENCES}

[1] G. Barbatis and A. Tertikas, On a class of Rellich inequalities, J. Comp. Appl. Math. 194 (2006), 156-172.

[2] W. Beckner, Geometric inequalities in Fourier analysis, Essays on Fourier Analysis in Honor of Elias M. Stein, Princeton University Press, 1995, pp. 36-68.

[3] W. Beckner, Pitt's inequality and the uncertainty principle, Proc. Amer. Math. Soc. 123 (1995), 1897-1905.

[4] W. Beckner, Sharp inequalities and geometric manifolds, J. Fourier Anal. Appl. 3 (1997), 825-836.

[5] S. Bochner, Harmonic analysis and the theory of probability, University of California Press, 1960.

[6] H. J. Brascamp, E. H. Lieb and J. M. Luttinger, A general rearrangement inequality for multiple integrals, J. Funct. Anal. 17 (1974), 227-237.

[7] S. Eilertsen, On weighted fractional integral inequalities, J. Funct. Anal. 185 (2001), 342-366.

[8] G. B. Folland and A. Sitaram, The uncertainty principle: a mathematical survey, J. Fourier Anal. Appl. 3 (1997), $207-238$.

[9] G. H. Hardy, J. E. Littlewood and G. Pólya, Inequalities, Cambridge University Press, 1952.

[10] H. R. Pitt, Theorems on Fourier series and power series, Duke Math. J. 3 (1937), 747-755.

[11] E. M. Stein, Singular integrals and differentiability properties of functions, Princeton University Press, 1970.

[12] E. M. Stein, Analytic continuation of group representations, Adv. Math. 4 (1970), 172-207.

[13] E. M. Stein and G. Weiss, Fractional integrals on n-dimensional Euclidean space, J. Math. Mech. 7 (1958), 503-514.

[14] A. Tertikas and N. B. Zographopoulos, Best constants in the Hardy-Rellich inequalities and related improvements, Adv. Math. (in press).

[15] E. T. Whittaker and G. N. Watson, A course of modern analysis, Cambridge University Press, 1927.

[16] D. Yafaev, Sharp constants in the Hardy-Rellich inequalities, J. Funct. Anal. 168 (1999), 121-144.

[17] A. Zygmund, Trigonometric series, Cambridge University Press, 1959.

Department of Mathematics, The University of Texas at Austin, 1 University Station C1200, Austin TX 78712-0257 USA

E-mail address: beckner@math.utexas.edu 